hep-ph/9904210

\title{
Atmospheric and Solar Neutrinos from Single Right-Handed Neutrino Dominance and $U(1)$ Family Symmetry
}

\author{
S. F. King \\ Department of Physics and Astronomy, University of Southampton, Southampton, \\ SO17 1BJ, U.K.
}

\begin{abstract}
We discuss a natural explanation of both neutrino mass hierarchies and large neutrino mixing angles, as required by the atmospheric neutrino data, in terms of a single righthanded neutrino giving the dominant contribution to the 23 block of the light effective neutrino matrix, and illustrate this mechanism in the framework of models with $U(1)$ family symmetries. Sub-dominant contributions from other right-handed neutrinos are required to give small mass splittings appropriate to the small angle MSW solution to the solar neutrino problem. We give general conditions for achieving this in the framework of $U(1)$ family symmetry models containing arbitrary numbers of righthanded neutrinos, and show how the resulting neutrino mass hierarchies and mixing angles may be expanded in terms of the Wolfenstein parameter.
\end{abstract}




\section{Introduction}

There is now strong evidence for atmospheric neutrino oscillations [1], [2] which confirms the earlier indications of the effect [3]. The most recent analyses of SuperKamiokande [1] involve the hypothesis of $\nu_{\mu} \rightarrow \nu_{\tau}$ oscillations with maximal mixing $\sin ^{2} 2 \theta_{23}=1$ and a mass splitting of $\Delta m_{23}^{2}=2.2 \times 10^{-3} \mathrm{eV}^{2}$. Using all their data sets analysed in different ways they quote $\sin ^{2} 2 \theta_{23}>0.82$ and a mass splitting of $5 \times 10^{-4} \mathrm{eV}^{2}<\Delta m_{23}^{2}<6 \times 10^{-3} \mathrm{eV}^{2}$ at $90 \%$ confidence level.

The evidence for solar neutrino oscillations is almost as strong [ []]. There are a panoply of experiments looking at different energy ranges, and the best fit to all of them has been narrowed down to two basic scenarios corresponding to either resonant oscillations $\nu_{e} \rightarrow \nu_{0}$ (where for example $\nu_{0}$ may be a linear combination of $\nu_{\mu}, \nu_{\tau}$ ) inside the Sun (MSW [5]) or "just-so" oscillations in the vacuum between the Sun and the Earth [6, []. There are three MSW fits and one vacuum oscillation fit:

(i) the small angle MSW solution is $\sin ^{2} 2 \theta_{12} \approx 5 \times 10^{-3}$ and $\Delta m_{12}^{2} \approx 5 \times 10^{-6} \mathrm{eV}^{2}$;

(ii) the large angle MSW solution is $\sin ^{2} 2 \theta_{12} \approx 0.76$ and $\Delta m_{12}^{2} \approx 1.8 \times 10^{-5} \mathrm{eV}^{2}$;

(iii) an additional MSW large angle solution exists with a lower probability [8];

(iv) The vacuum oscillation solution is $\sin ^{2} 2 \theta_{12} \approx 0.75$ and $\Delta m_{12}^{2} \approx 6.5 \times 10^{-11} \mathrm{eV}^{2}$ [8].

The standard model has zero neutrino masses, so any indication of neutrino mass is very exciting since it represents new physics beyond the standard model. In this paper we shall assume the see-saw mechanism and no light sterile neutrinos. The see-saw mechanism [9] implies that the three light neutrino masses arise from some heavy "right-handed neutrinos" $N_{R}^{p}$ (in general there can be $Z$ gauge singlets with $p=1, \ldots Z$ ) with a $Z \times Z$ Majorana mass matrix $M_{R R}^{p q}$ whose entries take values 
at or below the unification scale $M_{U} \sim 10^{16} \mathrm{GeV}$. The presence of electroweak scale Dirac mass terms $m_{L R}^{i p}\left(\right.$ a $3 \times Z$ matrix) connecting the left-handed neutrinos $\nu_{L}^{i}$ $(i=1, \ldots 3)$ to the right-handed neutrinos $N_{R}^{p}$ then results in a very light see-saw suppressed effective $3 \times 3$ Majorana mass matrix

$$
m_{L L}=m_{L R} M_{R R}^{-1} m_{L R}^{T}
$$

for the left-handed neutrinos $\nu_{L}^{i}$, which are the light physical degrees of freedom observed by experiment.

Not surprisingly, following the recent data, there has been a torrent of theoretical papers concerned with understanding how to extend the standard model in order to accomodate the atmospheric and solar neutrino data [10]. Perhaps the minimal extension of the standard model capable of accounting for the atmospheric neutrino data involves the addition of a single right-handed neutrino $N_{R}$ [11, 12]. This is a special case of the general see-saw model with $Z=1$, so that $M_{R R}$ is a trivial $1 \times 1$ matrix and $m_{L R}$ is a $3 \times 1$ column matrix where $m_{L R}^{T}=\left(\lambda_{\nu_{e}}, \lambda_{\nu_{\mu}}, \lambda_{\nu_{\tau}}\right) v_{2}$ with $v_{2}$ the vacuum expectation value of the Higgs field $H_{2}$ which is responsible for the neutrino Dirac masses, and the notation for the Yukawa couplings $\lambda_{i}$ indicates that we are in the charged lepton mass eigenstate basis $e_{L}, \mu_{L}, \tau_{L}$ with corresponding neutrinos $\nu_{e_{L}}, \nu_{\mu_{L}}, \nu_{\tau_{L}}$. Since $M_{R R}$ is trivially invertible the light effective mass matrix in Eq.1] in the $\nu_{e_{L}}, \nu_{\mu_{L}}, \nu_{\tau_{L}}$ basis is simply given by

$$
m_{L L}=\frac{m_{L R} m_{L R}^{T}}{M_{R R}}=\left(\begin{array}{lll}
\lambda_{\nu_{e}}^{2} & \lambda_{\nu_{e}} \lambda_{\nu_{\mu}} & \lambda_{\nu_{e}} \lambda_{\nu_{\tau}} \\
\lambda_{\nu_{e}} \lambda_{\nu_{\mu}} & \lambda_{\nu_{\mu}}^{2} & \lambda_{\nu_{\mu}} \lambda_{\nu_{\tau}} \\
\lambda_{\nu_{e}} \lambda_{\nu_{\tau}} & \lambda_{\nu_{\mu}} \lambda_{\nu_{\tau}} & \lambda_{\nu_{\tau}}^{2}
\end{array}\right) \frac{v_{2}^{2}}{M_{R R}} .
$$

The matrix in Eq.2 has vanishing determinant which implies a zero eigenvalue. Furthermore the submatrix in the 23 sector has zero determinant which implies a second zero eigenvalue associated with this sector. In order to account for the SuperKamiokande data we assumed [11]:

$$
\lambda_{\nu_{e}} \ll \lambda_{\nu_{\mu}} \approx \lambda_{\nu_{\tau}}
$$


In the $\lambda_{\nu_{e}}=0$ limit the matrix in Eq.2 has zeros along the first row and column, and so clearly $\nu_{e}$ is massless, and the other two eigenvectors are simply

$$
\left(\begin{array}{l}
\nu_{0} \\
\nu_{3}
\end{array}\right)=\left(\begin{array}{ll}
c_{23} & -s_{23} \\
s_{23} & c_{23}
\end{array}\right)\left(\begin{array}{l}
\nu_{\mu} \\
\nu_{\tau}
\end{array}\right)
$$

where $t_{23}=\lambda_{\nu_{\mu}} / \lambda_{\nu_{\tau}}$, with $\nu_{0}$ being massless, due to the vanishing of the determinant of the 23 submatrix and $\nu_{3}$ having a mass $m_{\nu_{3}}=\left(\lambda_{\nu_{\mu}}^{2}+\lambda_{\nu_{\tau}}^{2}\right) v_{2}^{2} / M_{R R}$. The SuperKamiokande data is accounted for by choosing the parameters such that $t_{23} \sim 1$ and $m_{\nu_{3}} \sim 5 \times 10^{-2} \mathrm{eV}$. In this approximation the atmospheric neutrino data is then consistent with $\nu_{\mu} \rightarrow \nu_{\tau}$ oscillations via two state mixing, between $\nu_{3}$ and $\nu_{0}$. Note how the single right-handed neutrino coupling to the 23 sector implies vanishing determinant of the 23 submatrix. This provides a natural explanation of both large 23 mixing angles and a hierarchy of neutrino masses in the 23 sector at the same time |11].

In order to account for the solar neutrino data a small mass perturbation is required to lift the massless degeneracy of the two neutrinos $\nu_{0}, \nu_{e}$. In our original approach [11] 凹e introduced additional right-handed neutrinos in order to provide a subdominant contribution to the effective mass matrix in Eq.2. To be precise we assumed a single dominant right-handed neutrino below the unification scale, with additional right-handed neutrinos at the unification scale which lead to subdominant contributions to the effective neutrino mass matrix. By appealing to quark and lepton mass hierarchy we assumed that the additional subdominant right-handed neutrinos generate a contribution $m_{\nu_{\tau}} \approx m_{t}^{2} / M_{U} \approx 2 \times 10^{-3} \mathrm{eV}$, where $m_{t}$ is the top quark mass. The effect of this is to give a mass perturbation to the 33 component of the mass matrix in Eq.2, which results in $\nu_{0}$ picking up a small mass, through its $\nu_{\tau}$ component, while $\nu_{e}$ remains massless. Solar neutrino oscillations then arise from $\nu_{e} \rightarrow \nu_{0}$ with

\footnotetext{
${ }^{1}$ Another approach $[12$ which does not rely on additional right-handed neutrinos is to use SUSY radiative corrections so that the one-loop corrected neutrino masses are not zero but of order $10^{-5}$ $\mathrm{eV}$ suitable for the vacuum oscillation solution.
} 
the mass splitting in the right range for the small angle MSW solution, controlled by a small mixing angle $\theta_{12} \approx \lambda_{\nu_{e}} / \sqrt{\lambda_{\nu_{\mu}}^{2}+\lambda_{\nu_{\tau}}^{2}}$. The main prediction of this scheme is of the neutrino oscillation $\nu_{e} \rightarrow \nu_{3}$ with a mass difference $\Delta m_{13}^{2} \approx \Delta m_{23}^{2}$ determined by the Super-Kamiokande data and a mixing angle $\theta_{13} \approx \theta_{12}$ determined by the small angle MSW solution. Such oscillations may be observable at the proposed long baseline experiments via $\nu_{3} \rightarrow \nu_{e}$ which implies $\nu_{\mu} \rightarrow \nu_{e}$ oscillations with $\sin ^{2} 2 \theta \approx 5 \times 10^{-3}$ (the small MSW angle) and $\Delta m^{2} \approx 2.2 \times 10^{-3} \mathrm{eV}^{2}$ (the Super-Kamiokande square mass difference).

It should be clear from the foregoing discussion that the motivation for single righthanded neutrino dominance (SRHND) is that the determinant of the 23 submatrix of Eq.2 approximately vanishes, leading to a natural explanation of both large neutrino mixing angles and hierarchical neutrino masses in the 23 sector at the same time [11. Although the explicit example of SRHND above was based on one of the right-handed neutrinos being lighter than the others, it is clear that the idea of SRHND is more general than this. In the present paper we shall define SRHND more generally as the requirement that a single right-handed neutrino gives the dominant contribution to the 23 submatrix of the light effective neutrino mass matrix. We shall propose SRHND as a general requirement and address the following two questions:

1. What are the general conditions under which SRHND in the 23 block can arise and how can we quantify the contribution of the sub-dominant right-handed neutrinos which are responsible for breaking the massless degeneracy, and allowing the small angle MSW solution?

2. How can we understand the pattern of neutrino Yukawa couplings in Eq.3 where the assumed equality $\lambda_{\nu_{\mu}} \approx \lambda_{\nu_{\tau}}$ is apparently at odds with the hierarchical Yukawa couplings in the quark and charged lepton sector?

In order to address the two questions above we shall discuss SRHND in the context of a $U(1)$ family symmetry. In fact neutrino masses and mixing angles have already 
been studied in the context of $U(1)$ family symmetry models but in the models that exist to date either SRHND is not present at all [13], [14], or where it is present its presence has apparently gone unnoticed [15]. 2] Where there is no SRHND, either the contribution to the 23 mixing angles coming from the neutrino matrix are small [13], or the 23 neutrino mass hierarchy is not described by the Wolfenstein expansion parameter [14]. Where the 23 neutrino mass hierarchies are described by the Wolfenstein expansion parameter and large 23 mixing angles naturally arise [15], we shall show that the physical reason why these models are successful is that a single right-handed neutrino is giving the dominant contribution to the 23 submatrix of $m_{L L}$. We shall give general conditions that theories with $U(1)$ family symmetry must satisfy in order to have SRHND and show that the models in 150 satisfy these conditions.

\section{$2 \quad$ MSSM with $Z$ Right-handed Neutrinos}

To fix the notation, we assume the Yukawa terms of the minimal supersymmetric standard model (MSSM) augmented by $Z$ right-handed neutrinos,

$$
\begin{array}{r}
\mathcal{L}_{y u k}=\epsilon_{a b}\left[-Y_{i j}^{u} H_{u}^{a} Q_{i}^{b} U_{j}^{c}+Y_{i j}^{d} H_{d}^{a} Q_{i}^{b} D_{j}^{c}+Y_{i j}^{e} H_{d}^{a} L_{i}^{b} E_{j}^{c}-Y_{i p}^{\nu} H_{u}^{a} L_{i}^{b} N_{p}^{c}+\frac{1}{2} M_{R R}^{p q} N_{p}^{c} N_{q}^{c}\right] \\
+H . c .
\end{array}
$$

where $\epsilon_{a b}=-\epsilon_{b a}, \epsilon_{12}=1$, and the remaining notation is standard except that the $Z$ right-handed neutrinos $N_{R}^{p}$ have been replaced by their CP conjugates $N_{p}^{c}$ with $p, q=1, \ldots, Z$. When the two Higgs doublets get their vacuum expectation values

\footnotetext{
${ }^{2}$ We should point out that the condition of the approximately vanishing subdeterminant was first clearly stated in ref. [15]. However all the actual examples presented there correspond to a single right-handed neutrino giving the dominant contribution to the 23 block of the effective neutrino mass matrix, which is essentially the mechanism first proposed in ref. [11]. Also note that SRHND has very recently been applied to an $S U(2)$ family symmetry model 16$]$.
} 
$(\mathrm{VEVS})<H_{u}^{2}>=v_{2},<H_{d}^{1}>=v_{1}$ with $\tan \beta \equiv v_{2} / v_{1}$ we find the terms

$$
\mathcal{L}_{y u k}=v_{2} Y_{i j}^{u} U_{i} U_{j}^{c}+v_{1} Y_{i j}^{d} D_{i} D_{j}^{c}+v_{1} Y_{i j}^{e} E_{i} E_{j}^{c}+v_{2} Y_{i p}^{\nu} N_{i} N_{p}^{c}+\frac{1}{2} M_{R R}^{p q} N_{p}^{c} N_{q}^{c}+H . c .
$$

Replacing CP conjugate fields we can write in a matrix notation

$$
\mathcal{L}_{y u k}=\bar{U}_{L} v_{2} Y^{u} U_{R}+\bar{D}_{L} v_{1} Y^{d} D_{R}+\bar{E}_{L} v_{1} Y^{e} E_{R}+\bar{N}_{L} v_{2} Y^{\nu} N_{R}+\frac{1}{2} N_{R}^{T} M_{R R} N_{R}+H . c .
$$

where we have assumed that all the masses and Yukawa couplings are real and written $Y^{\star}=Y$. The diagonal mass matrices are given by the following unitary transformations

$$
\begin{array}{r}
v_{2} Y_{\text {diag }}^{u}=V_{u L} v_{2} Y^{u} V_{u R}^{\dagger}=\operatorname{diag}\left(\mathrm{m}_{\mathrm{u}}, \mathrm{m}_{\mathrm{c}}, \mathrm{m}_{\mathrm{t}}\right), \\
v_{1} Y_{\text {diag }}^{d}=V_{d L} v_{1} Y^{d} V_{d R}^{\dagger}=\operatorname{diag}\left(\mathrm{m}_{\mathrm{d}}, \mathrm{m}_{\mathrm{s}}, \mathrm{m}_{\mathrm{b}}\right), \\
v_{1} Y_{\text {diag }}^{e}=V_{e L} v_{1} Y^{e} V_{e R}^{\dagger}=\operatorname{diag}\left(\mathrm{m}_{\mathrm{e}}, \mathrm{m}_{\mu}, \mathrm{m}_{\tau}\right), \\
M_{R R}^{\text {diag }}=\Omega_{R R} M_{R R} \Omega_{R R}^{\dagger}=\operatorname{diag}\left(\mathrm{M}_{\mathrm{R} 1}, \ldots, \mathrm{M}_{\mathrm{RZ}}\right),
\end{array}
$$

where the unitary transformations are also orthogonal. From Eq.1 the light effective left-handed Majorana neutrino mass matrix is

$$
m_{L L}=v_{2}^{2} Y_{\nu} M_{R R}^{-1} Y_{\nu}^{T}
$$

Having constructed the light Majorana mass matrix it must then be diagonalised by unitary transformations,

$$
m_{L L}^{\operatorname{diag}}=V_{\nu L} m_{L L} V_{\nu L}^{\dagger}=\operatorname{diag}\left(\mathrm{m}_{\nu_{1}}, \mathrm{~m}_{\nu_{2}}, \mathrm{~m}_{\nu_{3}}\right) .
$$

The CKM matrix is given by

$$
V_{C K M}=V_{u L} V_{d L}^{\dagger}
$$

and its leptonic analogue is the MNS matrix 17

$$
V_{M N S}=V_{\nu L} V_{e L}^{\dagger}
$$




\section{Wolfenstein Expansions}

The Wolfenstein parametrisation of the CKM matrix yields the approximate form 18 :

$$
V_{C K M} \sim\left(\begin{array}{lll}
1 & \lambda & \lambda^{3} \\
\lambda & 1 & \lambda^{2} \\
\lambda^{3} & \lambda^{2} & 1
\end{array}\right)
$$

where $\lambda \approx V_{u s} \approx 0.22$. The horizontal quark and lepton mass ratios may similarly be expanded in terms of the Wolfenstein parameter: [

$$
\frac{m_{u}}{m_{t}} \sim \lambda^{8}, \frac{m_{c}}{m_{t}} \sim \lambda^{4}, \frac{m_{d}}{m_{b}} \sim \lambda^{4}, \frac{m_{s}}{m_{b}} \sim \lambda^{2}, \frac{m_{e}}{m_{\tau}} \sim \lambda^{4}, \frac{m_{\mu}}{m_{\tau}} \sim \lambda^{2} .
$$

Assuming the MSSM the vertical quark and lepton mass ratios at $M_{U}$ are

$$
\frac{m_{b}}{m_{t}} \sim \lambda^{3}, \frac{m_{b}}{m_{\tau}} \sim 1 .
$$

Assuming that $V_{C K M} \sim V_{u L} \sim V_{d L}$, and the diagonal elements of the Yukawa matrices are of the same order as the eigenvalues:f

$$
Y^{u} \sim\left(\begin{array}{ccc}
\lambda^{8} & \lambda^{5} & \lambda^{3} \\
- & \lambda^{4} & \lambda^{2} \\
- & - & 1
\end{array}\right), \quad Y^{d} \sim\left(\begin{array}{ccc}
\lambda^{4} & \lambda^{3} & \lambda^{3} \\
- & \lambda^{2} & \lambda^{2} \\
- & - & 1
\end{array}\right) \lambda^{n}
$$

where $\tan \beta \sim \lambda^{n-3}$. Note that the CKM matrix only gives information about the upper triangular parts of the quark Yukawa matrices.

The MNS matrix is less well determined, but Super-Kamiokande tells us that $\theta_{23} \sim 1$ and the small angle MSW solution implies $\theta_{12} \sim \lambda^{2}$. In addition for $\Delta m^{2}>$ $9 \times 10^{-4} \mathrm{eV}^{2}$ (i.e. over most of the atmospheric range) CHOOZ [19] fails to observe $\nu_{e} \rightarrow \nu_{3}$ and excludes $\sin ^{2} 2 \theta_{13}>0.18$ or $\theta_{13}>0.22$. Hence CHOOZ allows $\theta_{13} \leq \lambda^{2}$. If we assume for the sake of argument that $\theta_{13} \sim \lambda^{2}$ (recall that this is a prediction of SRHND which follows from Eqs.(2 and (3) then $V_{M N S}$ is given by:

$$
V_{M N S} \sim\left(\begin{array}{lll}
1 & \lambda^{2} & \lambda^{2} \\
\lambda^{2} & 1 & 1 \\
\lambda^{2} & 1 & 1
\end{array}\right)
$$

\footnotetext{
${ }^{3}$ We follow the expansions in ref.[14] even though $\frac{m_{e}}{m_{\tau}} \sim \lambda^{5}$ is a better fit.

${ }^{4}$ Again this is similar to ref. [14] except that we allow a more general $\tan \beta$ dependence
} 
Then, in a similar way to the quarks, assuming that $V_{M N S} \sim V_{\nu L} \sim V_{e L}$, and the diagonal elements of the charged lepton matrix are of the same order as the eigenvalues we deduce

$$
Y^{e} \sim\left(\begin{array}{ccc}
\lambda^{4} & \lambda^{4} & \lambda^{2} \\
- & \lambda^{2} & 1 \\
- & - & 1
\end{array}\right) \lambda^{n} .
$$

The same argument applied to $m_{L L}$ runs into trouble because the hierarchy between the second and third eigenvalues is apparently not consistent with $\theta_{23} \sim 1$. To be precise Super-Kamiokande tells us that $m_{\nu_{3}} \approx 5 \times 10^{-2} \mathrm{eV}$, and small angle MSW tells us that $m_{\nu_{2}} \approx 2 \times 10^{-3} \mathrm{eV}$, hence

$$
\frac{m_{\nu_{2}}}{m_{\nu_{3}}} \sim \lambda^{2}
$$

The problem is how to generate such a hierarchy in the presence of large neutrino mixing angles. Note that this problem can be avoided for the charged lepton matrix in Eq.18 due to the undetermined 32 element which can be small, but for the symmetric neutrino matrix it is a problem. Fortunately the solution is provided by SRHND which implies that $m_{L L}$ is given from Eqs.2 and 3 as:

$$
m_{L L} \sim\left(\begin{array}{ccc}
\lambda^{4} & \lambda^{2} & \lambda^{2} \\
\lambda^{2} & 1 & 1 \\
\lambda^{2} & 1 & 1
\end{array}\right) m_{\nu_{3}}
$$

It is clear that SRHND leads to the prediction

$$
\frac{m_{\nu_{1}}}{m_{\nu_{3}}} \sim \lambda^{4}
$$

in addition to the previously mentioned prediction $\theta_{13} \sim \lambda^{2}$. The key to obtaining the hierarchy in Eq.19 from Eq.20 is the requirement that the determinant of the 23 submatrix must vanish to order $\lambda^{2}$. Since this subdeterminant naturally vanishes for a single right-handed neutrino coupling to the 23 sector, as in Eq.2, all that is required is for the subdominant right-handed neutrino to generate a perturbation to the masses in the 23 sector which are of order $\lambda^{2}$ smaller than the leading contribution. We shall now discuss how this can come about in the framework of theories with broken $U(1)$ family symmetry. 


\section{$4 \quad \mathrm{U}(1)$ Family Symmetry}

The idea of accounting for the fermion mass spectrum via a broken family symmetry has a long history [20], [21]. For definiteness we shall focus on a particular class of model based on a single pseudo-anomalous $U(1)_{X}$ gauged family symmetry [22]. We assume that the $U(1)_{X}$ is broken by the equal VEVs of two MSSM singlets $\theta, \bar{\theta}$ which have vector-like charges \pm 1 [22]. Theories in which the $U(1)_{X}$ is broken by a chiral MSSM singlet $\chi$ which has charge of one sign only, say +1 , have also been proposed [23], 24]. In all these cases the $U(1)_{X}$ has anomalies in the effective low energy theory below $M_{U}$ but these are compensated by string theory effects at $M_{U}$ and the Green-Schwartz mechanism [25] provides a dimension-five interaction term, whose structure demands a specific pattern among the anomaly coefficients [22]:

$$
A\left(S U(3)_{c}^{2} U(1)_{X}\right): A\left(S U(2)_{L}^{2} U(1)_{X}\right): A\left(U(1)_{Y}^{2} U(1)_{X}\right)=1: 1: 5 / 3
$$

The $U(1)_{X}$ breaking scale is set by $\langle\theta\rangle=\langle\bar{\theta}\rangle$ where the VEVs arise from a Green-Schwartz computable Fayet-Illiopoulos D-term which determines these VEVs to be one or two orders of magnitude below $M_{U}$. Additional exotic matter which exists in vector-like pairs with opposite charges $\pm X_{i}$ at a heavy mass scale $M_{V}$ (generated by the VEVs of yet more singlets) allows the Wolfenstein parameter to be generated by the ratio 22

$$
\frac{<\theta>}{M_{V}}=\frac{<\bar{\theta}>}{M_{V}}=\lambda \approx 0.22
$$

The idea is that at tree-level the $U(1)_{X}$ family symmetry only permits third family Yukawa couplings (e.g. the top quark Yukawa coupling). Smaller Yukawa couplings are generated effectively from higher dimension non-renormalisable operators corresponding to insertions of $\theta$ and $\bar{\theta}$ fields and hence to powers of the expansion parameter in Eq.23, which we have identified with the Wolfenstein parameter. The number of powers of the expansion parameter is controlled by the $U(1)_{X}$ charge of the particular 


\section{MSSM operator. 田}

The MSSM fields $Q_{i}, U_{j}^{c}, D_{j}^{c}, L_{i}, E_{j}^{c}, H_{u}, H_{d}$ are assigned $U(1)_{X}$ charges $q_{i}, u_{j}, d_{j}$, $l_{i}, e_{j}, h_{u}, h_{d}$ consistent with Eq.22. This restricts the physical values of the charges which we are permitted to assign. 0 We do not impose any restriction on the $Z$ right-handed MSSM singlet neutrinos $N_{p}^{c}$ which therefore have unconstrained charges $n_{p}$. We shall suppose that the right-handed neutrino Majorana mass matrix $M_{R R}$ arises from the VEV of another MSSM singlet $\Sigma$ with charge $\sigma$ [13]. The anomaly restriction means that there must exist a physical basis where the Higgs charges are equal and opposite, $h_{u}=-h_{d}$ in order to cancel their contributions to the anomalies, and gives a zero charge to the $\mu H_{u} H_{d}$ term. The other operators in Eq. general have non-zero charges and from Eqs.23, the associated Yukawa couplings and Majorana mass terms may then be expanded in powers of the Wolfenstein parameter,

$$
\begin{aligned}
& Y_{i j}^{u} \sim \lambda^{\left|q_{i}+u_{j}+h_{u}\right|}, \quad Y_{i j}^{d} \sim \lambda^{\left|q_{i}+d_{j}+h_{d}\right|}, \quad Y_{i j}^{e} \sim \lambda^{\left|l_{i}+e_{j}+h_{d}\right|}, \\
& Y_{i p}^{\nu} \sim \lambda^{\left|l_{i}+n_{p}+h_{u}\right|}, \quad M_{R R}^{p q} \sim \lambda^{\left|n_{p}+n_{q}+\sigma\right|}<\Sigma>.
\end{aligned}
$$

In the physical basis of charges discussed so far the quarks and leptons must contribute to the anomalies in the ratios in Eq.22. A corollary of this is that the physical charges are related to traceless charges (denoted by primes) by two flavourindependent $S U(5)$ shifts $\Delta t \equiv \Delta q=\Delta u=\Delta e$ and $\Delta f \equiv \Delta l=\Delta d[22]$ :

$$
q_{i}^{\prime}=q_{i}+\Delta t, \quad u_{i}^{\prime}=u_{i}+\Delta t, \quad e_{i}^{\prime}=e_{i}+\Delta t, \quad l_{i}^{\prime}=l_{i}+\Delta f, \quad d_{i}^{\prime}=d_{i}+\Delta f,
$$

\footnotetext{
${ }^{5}$ Of course this simple picture may in reality be more complicated if several different vector mass scales are assumed, and taking into account the order one dimensionless couplings involving different $\theta$ and $\bar{\theta}$ fields coupling the MSSM fields to the heavy vector matter. By making various dynamical assumptions it is possible to generate several different expansion parameters which may be in expanded non-integer powers [13]. It is also possible to introduce several $U(1)$ symmetries, such as a model recently proposed based on a family-independent pseudo-anomalous $U(1)_{X}$ symmetry together with two further anomaly-free but family-dependent $U(1)$ symmetries [14]. For our purposes here it is sufficient to assume a single $U(1)_{X}$ family symmetry with the single Wolfenstein expansion parameter in Eq.23 raised to integer powers.

${ }^{6}$ This restriction may be relaxed by assuming that the heavy vector matter has $X_{i}$ charges chosen to cancel the anomalies, but we prefer instead to regard this as a welcome constraint on the charges. We shall, however, allow heavy MSSM singlets with arbitrary charges to cancel $U(1)_{X}^{3}$ anomalies.
} 
It is possible to absorb the $S U(5)$ shifts into the Higgs charges by defining

$$
h_{u}^{\prime} \equiv h_{u}-2 \Delta t, \quad h_{d}^{\prime} \equiv h_{d}-\Delta t-\Delta f
$$

so that

$$
q_{i}+u_{j}+h_{u}=q_{i}^{\prime}+u_{j}^{\prime}+h_{u}^{\prime}, \quad q_{i}+d_{j}+h_{d}=q_{i}^{\prime}+d_{j}^{\prime}+h_{d}^{\prime}, \quad l_{i}+e_{j}+h_{d}=l_{i}^{\prime}+e_{j}^{\prime}+h_{d}^{\prime} .
$$

The couplings in Eq.24 may then be equivalently expanded in terms of primed charges. Tracelessness implies that the first family charges may be eliminated

$$
q_{1}^{\prime}=-q_{2}^{\prime}-q_{3}^{\prime}, \quad u_{1}^{\prime}=-u_{2}^{\prime}-u_{3}^{\prime}, \quad d_{1}^{\prime}=-d_{2}^{\prime}-d_{3}^{\prime}, \quad l_{1}^{\prime}=-l_{2}^{\prime}-l_{3}^{\prime}, \quad e_{1}^{\prime}=-e_{2}^{\prime}-e_{3}^{\prime} .
$$

Since the 33 component of the Yukawa matrices are either renormalisable or related to $\tan \beta$ dependent integers we can eliminate the primed Higgs charges using

$$
h_{u}^{\prime}=-q_{3}^{\prime}-u_{3}^{\prime}, \quad h_{d}^{\prime}=n_{d}-q_{3}^{\prime}-d_{3}^{\prime}=n_{e}-l_{3}^{\prime}-e_{3}^{\prime}
$$

Using Eqs.28, 29, 30 the Yukawa matrices in 24 may then be expressed as

$$
\begin{aligned}
Y^{u} \sim\left(\begin{array}{lll}
\lambda^{\left|\gamma_{u}+\delta_{u}\right|} & \lambda^{\left|\gamma_{u}+\beta_{u}\right|} & \lambda^{\left|\gamma_{u}\right|} \\
\lambda^{\left|\alpha_{u}+\delta_{u}\right|} & \lambda^{\left|\alpha_{u}+\beta_{u}\right|} & \lambda^{\left|\alpha_{u}\right|} \\
\lambda^{\left|\delta_{u}\right|} & \lambda^{\left|\beta_{u}\right|} & 1
\end{array}\right), & Y^{d} \sim\left(\begin{array}{lll}
\lambda^{\left|\gamma_{d}+\delta_{d}+n_{d}\right|} & \lambda^{\left|\gamma_{d}+\beta_{d}+n_{d}\right|} & \lambda^{\left|\gamma_{d}+n_{d}\right|} \\
\lambda^{\left|\alpha_{d}+\delta_{d}+n_{d}\right|} & \lambda^{\left|\alpha_{d}+\beta_{d}+n_{d}\right|} & \lambda^{\left|\alpha_{d}+n_{d}\right|} \\
\lambda^{\left|\delta_{d}+n_{d}\right|} & \lambda^{\left|\beta_{d}+n_{d}\right|} & \lambda^{\left|n_{d}\right|}
\end{array}\right), \\
& Y^{e} \sim\left(\begin{array}{lll}
\lambda^{\left|\gamma_{e}+\delta_{e}+n_{e}\right|} & \lambda^{\left|\gamma_{e}+\beta_{e}+n_{e}\right|} & \lambda^{\left|\gamma_{e}+n_{e}\right|} \\
\lambda^{\left|\alpha_{e}+\delta_{e}+n_{e}\right|} & \lambda^{\left|\alpha_{e}+\beta_{e}+n_{e}\right|} & \lambda^{\left|\alpha_{e}+n_{e}\right|} \\
\lambda^{\left|\delta_{e}+n_{e}\right|} & \lambda^{\left|\beta_{e}+n_{e}\right|} & \lambda^{\left|n_{e}\right|}
\end{array}\right),
\end{aligned}
$$

where

$$
\begin{aligned}
& \alpha_{u}=\alpha_{d}=q_{2}^{\prime}-q_{3}^{\prime}, \quad \alpha_{e}=l_{2}^{\prime}-l_{3}^{\prime}, \\
& \beta_{u}=u_{2}^{\prime}-u_{3}^{\prime}, \quad \beta_{d}=d_{2}^{\prime}-d_{3}^{\prime}, \quad \beta_{e}=e_{2}^{\prime}-e_{3}^{\prime}, \\
& \gamma_{u}=\gamma_{d}=-q_{2}^{\prime}-2 q_{3}^{\prime}, \quad \gamma_{e}=-l_{2}^{\prime}-2 l_{3}^{\prime}, \\
& \delta_{u}=-u_{2}^{\prime}-2 u_{3}^{\prime}, \quad \delta_{d}=-d_{2}^{\prime}-2 d_{3}^{\prime}, \quad \delta_{e}=-e_{2}^{\prime}-2 e_{3}^{\prime} .
\end{aligned}
$$

The above analysis applies quite generally to any theory based on a single pseudoanomalous $U(1)_{X}$ gauged family symmetry. However the quark and lepton charges may be constrained by imposing unification constraints on the theory. For example: 
- $S U(5)$ unification implies $l_{i}=d_{i}, q_{i}=u_{i}=e_{i}$ but allows $Z$ arbitrary righthanded neutrino charges $n_{p}$.

- $S U(2)_{R}$ gauge symmetry implies that $Z=3$ with $n_{i}=e_{i}$ and $d_{i}=u_{i}$.

- Left-right symmetry is stronger than $S U(2)_{R}$ and implies $n_{i}=e_{i}=l_{i}$ and $d_{i}=u_{i}=q_{i}$.

- Pati-Salam $S U(4) \times S U(2)_{L} \times S U(2)_{R}$ implies $l_{i}=q_{i}$ and $u_{i}=d_{i}=e_{i}=n_{i}$.

- $S O(10)$ unification implies $l_{i}=d_{i}=q_{i}=u_{i}=e_{i}=n_{i}$

- Trinification $S U(3)^{3}$ implies $u_{i}=d_{i}, l_{i}=e_{i}=n_{i}$ and unconstrained $q_{i}$

- Flipped $S U(5) \times U(1)$ implies $q_{i}=d_{i}=n_{i}, u_{i}=l_{i}$ and unconstrained $e_{i}$

As discussed in ref. [13] these examples are difficult to reconcile with the data without either appealing to group theoretical Clebsch relations or carefully chosen dynamical assumptions. 7 We shall therefore not impose such gauge unification constraints here but instead consider the general case in Eqs.31.

By comparing Eqs.13, to Eqs.16, 18 suitable choices of the integers $\alpha_{a}, \beta_{a}, \gamma_{a}, \delta_{a}$, (where $a=u, d, e$ ) can readily be deduced. Note especially that $m_{b} / m_{\tau} \sim 1$ implies

$$
n=\left|n_{e}\right|=\left|n_{d}\right|, \quad \tan \beta \sim \lambda^{n-3} .
$$

It is straightforward to scan over all the possible positive and negative integers $\alpha_{a}, \beta_{a}, \gamma_{a}, \delta_{a}, n_{a}$ to find acceptable Yukawa matrices from Eqs.31. For example a special case is when $\alpha_{a}, \beta_{a}, \gamma_{a}, \delta_{a}, n_{a}$ are all positive definite integers [14. In this case from Eqs.16, 18, 31 we find $\alpha_{u}=\alpha_{d}=2, \alpha_{e}=0, \beta_{u}=2, \beta_{d}=0, \beta_{e}=2, \gamma_{u}=\gamma_{d}=3$,

\footnotetext{
${ }^{7}$ Note that $S U(5)$ automatically guarantees Green-Schwartz anomaly cancellation for any choice of charges.

${ }^{8}$ The $S U(3)^{3}$ model discussed there looks the most natural.
} 
$\gamma_{e}=2, \delta_{u}=5, \delta_{d}=1, \delta_{e}=2, n_{e}=n_{d}=n$. f The Yukawa matrices are then fully specified in this example, up to a $\tan \beta$ dependence:

$$
Y^{u} \sim\left(\begin{array}{ccc}
\lambda^{8} & \lambda^{5} & \lambda^{3} \\
\lambda^{7} & \lambda^{4} & \lambda^{2} \\
\lambda^{5} & \lambda^{2} & 1
\end{array}\right), \quad Y^{d} \sim\left(\begin{array}{ccc}
\lambda^{4} & \lambda^{3} & \lambda^{3} \\
\lambda^{3} & \lambda^{2} & \lambda^{2} \\
\lambda & 1 & 1
\end{array}\right) \lambda^{n}, \quad Y^{e} \sim\left(\begin{array}{ccc}
\lambda^{4} & \lambda^{4} & \lambda^{2} \\
\lambda^{2} & \lambda^{2} & 1 \\
\lambda^{2} & \lambda^{2} & 1
\end{array}\right) \lambda^{n} .
$$

Given $\alpha_{a}, \beta_{a}, \gamma_{a}, \delta_{a}, n_{a}$ above and using Eqs.29, 30, 32 we find the following traceless:

$$
\begin{aligned}
& q_{i}^{\prime}=\frac{1}{3}(4,1,-5), \quad u_{i}^{\prime}=\frac{1}{3}(8,-1,-7), \quad d_{i}^{\prime}=\frac{1}{3}(2,-1,-1) \\
& l_{i}^{\prime}=\frac{1}{3}(4,-2,-2), \quad e_{i}^{\prime}=\frac{1}{3}(2,2,-4), \quad h_{u}^{\prime}=4, \quad h_{d}^{\prime}=2+n
\end{aligned}
$$

The physical (unprimed) charges are by definition those which lead to the Higgs charges satisfying $h_{u}=-h_{d}$. Eq.27 shows that there remains an ambiguity in the choice of Higgs charges and hence in $\Delta t, \Delta f$ which are two unknowns constrained by only one relation, namely $-3 \Delta t=h_{d}^{\prime}+h_{u}^{\prime}+\Delta f$. We can regard $\Delta f$ as being a completely free parameter whose choice specifies all the physical (unprimed) charges uniquely. For example we may set the Higgs charges to be zero by taking $10 t=-2$, $\Delta f=-n$ which enables the physical (unprimed) charges to be deduced from Eq.26. Other choices of $\Delta f$ will lead to different choices of physical charges.

\section{SRHND and U(1) Family Symmetry}

We now turn our attention to the neutrino sector, which is the main focus of this paper. Since the $Z$ right-handed neutrinos are not constrained by anomaly cancellation it is most convenient to work with physical (unprimed) charges as in Eq.25. $Y^{\nu}$

\footnotetext{
${ }^{9}$ Note that $n_{e}=n_{d}=n$ imposes the non-trivial constraint that $\alpha_{e}+\beta_{e}+\gamma_{e}+\delta_{e}=\alpha_{d}+\beta_{d}+\gamma_{d}+\delta_{d}$ which is satisfied here. If for example we had taken $\frac{m_{e}}{m_{\tau}} \sim \lambda^{5}$ it would not be satisfied.

${ }^{10}$ Note that in general both both $\Delta t$ and $\Delta f$ are non-zero and so the family symmetry $U(1)_{X}$ cannot be anomaly-free and is instead pseudo-anomalous [22].
} 
clearly depends on the combination of lepton and Higgs charges

$$
l_{i}+h_{u}=l_{i}^{\prime}+h_{u}^{\prime} / 3-2 h_{d}^{\prime} / 3-5 \Delta f / 3
$$

which is not fixed by the primed charges due to the remaining freedom in $\Delta f$. In dealing with the neutrino sector it is convenient to absorb the Higgs charge $h_{u}$ into the definition of the lepton charges $l_{i}$ so that Eq.25 becomes

$$
Y_{i p}^{\nu} \sim \lambda^{\left|l_{i}+n_{p}\right|}, \quad M_{R R}^{p q} \sim \lambda^{\left|n_{p}+n_{q}+\sigma\right|}<\Sigma>
$$

where the redefined $l_{i}$ are related to the traceless charges $l_{i}^{\prime}$ by arbitrary familyindependent shifts, and using Eq.35 may be written as:

$$
l_{i}=\left(2+l_{3}, l_{3}, l_{3}\right)
$$

where the numerical value of $l_{3}$ remains a free choice.

The light Majorana matrix may then be constructed from Eq.9 which we repeat below

$$
m_{L L}=v_{2}^{2} Y_{\nu} M_{R R}^{-1} Y_{\nu}^{T}
$$

If we were to assume positive definite values for $l_{i}+n_{p}$ and $n_{p}+n_{q}+\sigma$ then the modulus signs could be dropped and the right-handed neutrino charges $n_{p}$ would cancel when $m_{L L}$ is constructed from Eqs 38 and 36 [26]. The argument relies on the observation that if the modulus signs are dropped from Eq.36 one can always write

$$
\begin{aligned}
& Y^{\nu}=\operatorname{diag}\left(\lambda^{l_{1}}, \lambda^{l_{2}}, \lambda^{l_{3}}\right) Y_{D} \operatorname{diag}\left(\lambda^{n_{1}}, \ldots, \lambda^{n_{Z}}\right), \\
& M_{R R}=\operatorname{diag}\left(\lambda^{n_{1}}, \ldots, \lambda^{n_{Z}}\right) M_{M} \operatorname{diag}\left(\lambda^{n_{1}}, \ldots, \lambda^{n_{Z}}\right)
\end{aligned}
$$

where $Y_{D}$ and $M_{M}$ are democratic matrices. Inserting Eq.39 into Eq.9 the righthanded neutrino charges are seen to cancel. Such a cancellation would imply that every right-handed neutrino would contribute equally to every entry in $m_{L L}$ regardless of the right-handed neutrino charges. From the point of view of SRHND it is therefore 
important that such a cancellation does not take place, and so we shall require that at least some of the combinations $l_{i}+n_{p}$ and $n_{p}+n_{q}+\sigma$ take negative values. In such a case the choice of right-handed neutrino charges will play an important role in determining $m_{L L}$, and each particular choice of $n_{p}$ must be analysed separately.

At first sight the general case of $Z$ right-handed neutrinos with unconstrained charges $n_{p}$ leading to non-positive definite exponents in Eq.36 seems to make the determination of $m_{L L}$ an intractable problem. However we have already argued that the atmospheric neutrino data suggests SRHND in the 23 sector and this will lead to $m_{L L}$ of the form given in Eq.20. We shall now formulate the general conditions which will lead to SRHND in the 23 sector.

\subsection{One Right-handed Neutrino}

Let us first consider the case $Z=1$ where there is just a single right-handed neutrino, which for later convenience we shall refer to as $N_{3}^{c}$ with charge $n_{3}$. In this case Eq.36 becomes

$$
Y_{i 3}^{\nu} \sim \lambda^{\left|l_{i}+n_{3}\right|}, \quad M_{R R}^{33} \sim \lambda^{\left|2 n_{3}+\sigma\right|}<\Sigma>.
$$

Being a $1 \times 1$ matrix $M_{R R}^{33}$ is trivially inverted and we obtain from Eqs.38,

$$
m_{L L}^{i j} \sim \lambda^{\left|l_{i}+n_{3}\right|} \lambda^{\left|l_{j}+n_{3}\right|} \frac{v_{2}^{2}}{M_{R R}^{33}}
$$

which should be compared to Eq.2, where we identify प

$$
Y_{i 3}^{\nu} \sim \lambda^{\left|l_{i}+n_{3}\right|} \sim\left(\lambda_{\nu_{e}}, \lambda_{\nu_{\mu}}, \lambda_{\nu_{\tau}}\right)
$$

Then Eq.20 requires that

$$
\left|l_{2}+n_{3}\right|=\left|l_{3}+n_{3}\right|, \quad\left|l_{1}+n_{3}\right|-\left|l_{3}+n_{3}\right|=2
$$

\footnotetext{
${ }^{11}$ Even though the couplings in Eq.2 2 were defined in the diagonal charged lepton basis, the identification is still valid to a consistent order of the expansion parameter.
} 
If both $l_{2}+n_{3}$ and $l_{3}+n_{3}$ have the same sign (SS) then $l_{2}=l_{3}$, whereas if they have opposite signs (OS) then $l_{2}+l_{3}=-2 n_{3}$. Similarly if both $l_{1}+n_{3}$ and $l_{3}+n_{3}$ have the SS then $l_{1}-l_{3}=2$, whereas if they have OS then $l_{1}+l_{3}=2-2 n_{3}$. Interestingly the SS cases $l_{2}=l_{3}, l_{1}-l_{3}=2$ have already arisen in the example in Eq.35, which corresponds to $l_{i}$ charges in Eq.37. This is no surprise since it originates from the charged lepton Yukawa matrix in Eq.18 which follows from the assumption $V_{M N S} \sim V_{\nu L} \sim V_{e L}$ and the Super-Kamiokande data and the MSW solution.

To summarise, from Eqs.40, 41 and imposing Eq.43 the single right-handed neutrino included so far leads to

$$
m_{L L} \sim\left(\begin{array}{lll}
\lambda^{4} & \lambda^{2} & \lambda^{2} \\
\lambda^{2} & 1 & 1 \\
\lambda^{2} & 1 & 1
\end{array}\right) m_{\nu_{3}}
$$

where the atmospheric neutrino mass is given

$$
m_{\nu_{3}} \sim \lambda^{2\left|l_{3}+n_{3}\right|-\left|2 n_{3}+\sigma\right|} \frac{v_{2}^{2}}{<\Sigma>}
$$

With only a single right-handed neutrino $m_{L L}$ in Eq.44 has two zero eigenvalues, and a vanishing determinant of the 23 submatrix, as in Eq.2. In order to implement the small angle MSW solution we need to include the effect of subdominant right-handed neutrinos which break the massless degeneracy. SRHND requires that the elements in the 23 sector of Eq.4 must receive corrections of order $\lambda^{2}$ from the subdominant neutrinos so that the determinant of the 23 submatrix only approximately vanishes to this order leading to a small eigenvalue of order $\lambda^{2}$ and the desired mass hierarchy in Eq.19.

\subsection{Two Right-handed Neutrinos}

We now include a second right-handed neutrino $N_{2}^{c}$ with charge $n_{2}$, in addition to $N_{3}^{c}$ with charge $n_{3}$. With two right-handed neutrinos, $Z=2$, the heavy Majorana mass 
matrix from Eq.36 is

$$
M_{R R} \sim\left(\begin{array}{ll}
\lambda^{\left|2 n_{2}+\sigma\right|} & \lambda^{\left|n_{2}+n_{3}+\sigma\right|} \\
\lambda^{\left|n_{2}+n_{3}+\sigma\right|} & \lambda^{\left|2 n_{3}+\sigma\right|}
\end{array}\right)<\Sigma>
$$

For SRHND we clearly require $n_{2} \neq n_{3}$ to avoid the two right-handed neutrinos contributing democratically. More generally for SRHND we need to avoid large righthanded neutrino mixing angles. If we assume without loss of generality that $\lambda^{\left|2 n_{2}+\sigma\right|}>$ $\lambda^{\left|2 n_{3}+\sigma\right|}$, so that $N_{2}^{c}$ is heavier than $N_{3}^{c}$, then this implies

$$
\left|2 n_{2}+\sigma\right|<\left|2 n_{3}+\sigma\right|
$$

Then the small mixing angle requirement is

$$
\left|2 n_{2}+\sigma\right|<\left|n_{2}+n_{3}+\sigma\right|
$$

The lightest eigenvalue is of order the diagonal element provided

$$
\left|2 n_{2}+\sigma\right| \leq 2\left|n_{2}+n_{3}+\sigma\right|-\left|2 n_{3}+\sigma\right|
$$

Assuming all these conditions are met then $M_{R R}$ will be diagonalised by small angle rotations and have hierarchical eigenvalues set by the diagonal elements. As a first approximation we may drop the off-diagonal elements and write

$$
M_{R R} \approx \operatorname{diag}\left(M_{R 2}, M_{R 3}\right)
$$

where

$$
M_{R 2} \sim \lambda^{\left|2 n_{2}+\sigma\right|}<\Sigma>, \quad M_{R 3} \sim \lambda^{\left|2 n_{3}+\sigma\right|}<\Sigma>
$$

Then the light Majorana matrix is given by adding the separate contribution from each of the two right-handed neutrinos

$$
m_{L L}^{i j}=v_{2}^{2}\left(\frac{Y_{\nu}^{i 2} Y_{\nu}^{j 2}}{M_{R 2}}+\frac{Y_{\nu}^{i 3} Y_{\nu}^{j 3}}{M_{R 3}}\right)
$$

It is clear that the dominant contribution to a particular element of $m_{L L}$ will come from the right-handed neutrino which is at the same time the lightest, and couples 
the most strongly to left-handed neutrinos. Without loss of generality we have taken $N_{3}^{c}$ to be the lighter right-handed neutrino and to give the dominant contribution to the 23 block of $m_{L L}$ in Eq.41. We therefore write the subdominant contribution coming from the second right-handed neutrino $N_{2}^{c}$ as

$$
\delta m_{L L}^{i j}=\lambda^{\left|l_{i}+n_{2}\right|} \lambda^{\left|l_{j}+n_{2}\right|} \frac{v_{2}^{2}}{M_{R 2}}
$$

As discussed below Eq.44 we require:

$$
\frac{\delta m_{L L}^{33}}{m_{L L}^{33}}=\frac{\lambda^{2\left|l_{3}+n_{2}\right|}}{\lambda^{2\left|l_{3}+n_{3}\right|}} \frac{M_{R 3}}{M_{R 2}} \sim \lambda^{2}
$$

From Eqs. [1,

$$
\frac{M_{R 3}}{M_{R 2}} \sim \lambda^{\left|2 n_{3}+\sigma\right|-\left|2 n_{2}+\sigma\right|}
$$

so Eq.54 implies the condition

$$
2\left|l_{3}+n_{2}\right|-2\left|l_{3}+n_{3}\right|+\left|2 n_{3}+\sigma\right|-\left|2 n_{2}+\sigma\right|=2
$$

We already observed that the required MSW perturbation is

$$
\delta m_{L L}^{33} \sim \frac{v_{2}^{2}}{M_{U}}
$$

so we deduce

$$
\frac{M_{R 2}}{M_{U}} \sim \lambda^{2\left|l_{3}+n_{2}\right|}, \quad \frac{<\Sigma>}{M_{U}} \sim \lambda^{2\left|l_{3}+n_{2}\right|-\left|2 n_{2}+\sigma\right|}
$$

There is the further requirement that the powers of $\lambda$ occuring in $M_{R R}$ and $Y_{\nu}$ be either integer or half-integer. 13 By scanning over half-integer and integer values of $l_{3}, n_{2}, n_{3}, \sigma$ we find that there are no solutions which satisfy all the above constraints for integer powers of $\lambda$ in $M_{R R}$ and $Y_{\nu}$. ${ }^{3}$ However there are a large number of solutions involving half-integer powers of $\lambda$ in $M_{R R}$ and $Y_{\nu}$ (of course $m_{L L}$ in Eq.20 always

\footnotetext{
${ }^{12}$ In the case of half-integer powers this implies that the $\theta, \bar{\theta}$ fields which break the $U(1)_{X}$ symmetry must have charges $\pm 1 / 2$ and the expansion parameter in Eq.23 must be redefined so that $\frac{\langle\theta\rangle}{M_{V}}=$ $\frac{<\bar{\theta}>}{M_{Y}}=\lambda^{1 / 2}$, as in ref. [13].

${ }^{{ }^{13} \mathrm{I}}$ am grateful to Y. Nir (private communication) for pointing this out.
} 
involves integer powers of $\lambda$.) The condition in Eq.56 may be achieved in various ways with $N_{3}^{c}$ being lighter than $N_{2}^{c}$ by a factor, $M_{R 3} / M_{R 2} \sim \lambda^{\left|2 n_{3}+\sigma\right|-\left|2 n_{2}+\sigma\right|} \sim \lambda^{a}$, and the ratio of the Dirac couplings of $N_{2}^{c}, N_{3}^{c}$ to $L_{3}$ given by $\lambda^{2\left|l_{3}+n_{2}\right|-2\left|l_{3}+n_{3}\right|} \sim \lambda^{2-a}$, where $a>0$ is a positive integer. For example $l_{3}=-1 / 2, n_{2}=0, n_{3}=1, \sigma=0$ satisfies all the conditions with $a=2$ and $Y_{\nu}$ involving half-integer exponents. Further examples are listed in Table 1.

\subsection{Three Right-handed Neutrinos}

We now wish to extend the discussion to include three right-handed neutrinos $Z=3$, by introducing a third right-handed neutrino $N_{1}^{c}$ with charge $n_{1}$ in addition to the two already introduced above. Again we shall suppose that $N_{3}^{c}$ gives the dominant contribution to the 23 sector masses. As for the $Z=2$ case we require $n_{3} \neq n_{2}, n_{1}$, and we need to ensure that $N_{3}^{c}$ does not have large mixing angles in $M_{R R}$ in order to isolate it from the other right-handed neutrinos. This can be ensured by a sequence of conditions similar to Eqs.47, 48, 49. Then, after small angle rotations, $M_{R R}$ can be written in block diagonal form.

$$
M_{R R} \sim\left(\begin{array}{lll}
\lambda^{\left|2 n_{1}+\sigma\right|} & \lambda^{\left|n_{1}+n_{2}+\sigma\right|} & 0 \\
\lambda^{\left|n_{2}+n_{1}+\sigma\right|} & \lambda^{\left|2 n_{2}+\sigma\right|} & 0 \\
0 & 0 & \lambda^{\left|2 n_{3}+\sigma\right|}
\end{array}\right)<\Sigma>
$$

which is the analogue of Eq.50. The new feature of the $Z=3$ case compared to the $Z=2$ case is that there are now several possibilities for the structure of the upper $2 \times 2$ block in Eq.59 which are all consistent with SRHND, which are listed below.

"Diagonal dominated" corresponding to $\left|n_{1}+n_{2}+\sigma\right|>\min \left(\left|2 n_{1}+\sigma\right|,\left|2 n_{2}+\sigma\right|\right)$ :

$$
M_{R R}^{\text {upper }} \sim\left(\begin{array}{ll}
\lambda^{\left|2 n_{1}+\sigma\right|} & 0 \\
0 & \lambda^{\left|2 n_{2}+\sigma\right|}
\end{array}\right)<\Sigma>
$$

"Off-diagonal dominated" corresponding to $\left|n_{1}+n_{2}+\sigma\right|<\left|2 n_{1}+\sigma\right|,\left|2 n_{2}+\sigma\right|$ :

$$
M_{R R}^{\text {upper }} \sim\left(\begin{array}{ll}
0 & \lambda^{\left|n_{1}+n_{2}+\sigma\right|} \\
\lambda^{\left|n_{2}+n_{1}+\sigma\right|} & 0
\end{array}\right)<\Sigma>
$$




\begin{tabular}{ccccc}
\hline$l_{3}$ & $n_{2}$ & $n_{3}$ & $\sigma$ & $a$ \\
\hline-1 & $-1 / 2$ & 0 & 1 & 1 \\
\hline-1 & 0 & $1 / 2$ & 0 & 1 \\
\hline-1 & 0 & $1 / 2$ & $1 / 2$ & 1 \\
\hline-1 & 0 & $1 / 2$ & 1 & 1 \\
\hline-1 & $1 / 2$ & 1 & -1 & 1 \\
\hline-1 & $1 / 2$ & 1 & $-1 / 2$ & 1 \\
\hline-1 & $1 / 2$ & 1 & 0 & 1 \\
\hline-1 & $1 / 2$ & 1 & $1 / 2$ & 1 \\
\hline-1 & $1 / 2$ & 1 & 1 & 1 \\
\hline$-1 / 2$ & $-1 / 2$ & 0 & 1 & 1 \\
\hline$-1 / 2$ & 0 & $1 / 2$ & 0 & 1 \\
\hline$-1 / 2$ & 0 & $1 / 2$ & $1 / 2$ & 1 \\
\hline$-1 / 2$ & 0 & $1 / 2$ & 1 & 1 \\
\hline 0 & $-1 / 2$ & 0 & 1 & 1 \\
\hline 0 & $1 / 2$ & 0 & -1 & 1 \\
\hline $1 / 2$ & 0 & $-1 / 2$ & -1 & 1 \\
\hline $1 / 2$ & 0 & $-1 / 2$ & $-1 / 2$ & 1 \\
\hline $1 / 2$ & 0 & $-1 / 2$ & 0 & 1 \\
\hline $1 / 2$ & $1 / 2$ & 0 & -1 & 1 \\
\hline 1 & $-1 / 2$ & -1 & -1 & 1 \\
\hline 1 & $-1 / 2$ & -1 & $-1 / 2$ & 1 \\
\hline 1 & $-1 / 2$ & -1 & 0 & 1 \\
\hline 1 & $-1 / 2$ & -1 & $1 / 2$ & 1 \\
\hline 1 & $-1 / 2$ & -1 & 1 & 1 \\
\hline 1 & 0 & $-1 / 2$ & -1 & 1 \\
\hline 1 & 0 & $-1 / 2$ & $-1 / 2$ & 1 \\
\hline 1 & 0 & $-1 / 2$ & 0 & 1 \\
\hline 1 & $1 / 2$ & 0 & -1 & 1 \\
\hline$-1 / 2$ & 0 & 1 & 0 & 2 \\
\hline$-1 / 2$ & 0 & 1 & $1 / 2$ & 2 \\
\hline$-1 / 2$ & 0 & 1 & 1 & 2 \\
\hline 0 & $-1 / 2$ & $1 / 2$ & 1 & 2 \\
\hline 0 & $1 / 2$ & $-1 / 2$ & -1 & 2 \\
\hline $1 / 2$ & 0 & -1 & -1 & 2 \\
\hline $1 / 2$ & 0 & -1 & $-1 / 2$ & 2 \\
\hline $1 / 2$ & 0 & -1 & 0 & 2 \\
\hline 0 & $-1 / 2$ & 1 & 1 & 3 \\
\hline 0 & $1 / 2$ & -1 & -1 & 3 \\
\hline & & & & \\
\hline
\end{tabular}

Table 1: Simple $Z=2$ examples which satisfy all the conditions of SRHND given in the text. 
"Democratic" corresponding to $\left|n_{1}+n_{2}+\sigma\right|=\left|2 n_{1}+\sigma\right|=\left|2 n_{2}+\sigma\right|$ :

$$
M_{R R}^{u p p e r} \sim\left(\begin{array}{ll}
\lambda^{\left|2 n_{1}+\sigma\right|} & \lambda^{\left|n_{1}+n_{2}+\sigma\right|} \\
\lambda^{\left|n_{2}+n_{1}+\sigma\right|} & \lambda^{\left|2 n_{2}+\sigma\right|}
\end{array}\right)<\Sigma>
$$

In the "diagonal dominated" case after small angle rotations the light effective Majorana mass matrix in Eq. 38 may be calculated in the diagonal right-handed neutrino basis

$$
m_{L L}=v_{2}^{2} Y_{\nu} M_{R R}^{-1} Y_{\nu}^{T}=v_{2}^{2} Y_{\nu} \Omega_{R R}^{\dagger}\left(M_{R R}^{\text {diag }}\right)^{-1} \Omega_{R R} Y_{\nu}^{T}
$$

The advantage of working in a diagonal right-handed neutrino mass basis is that $\left(M_{R R}^{\text {diag }}\right)^{-1}=\operatorname{diag}\left(\mathrm{M}_{\mathrm{R} 1}^{-1}, \mathrm{M}_{\mathrm{R} 2}^{-1}, \mathrm{M}_{\mathrm{R} 3}^{-1}\right)$ so if we define $\tilde{Y}_{\nu} \equiv Y_{\nu} \Omega_{R R}^{\dagger}$ as the neutrino Yukawa matrix in the diagonal right-handed neutrino basis, then the effective light mass matrix elements are given from Eq.63 by

$$
m_{L L}^{i j}=\sum_{p=1}^{3} v_{2}^{2} \frac{\tilde{Y}_{\nu}^{i p} \tilde{Y}_{\nu}^{j p}}{M_{R p}}
$$

In this case $\Omega_{R R}$ involves small angle rotations and so $\tilde{Y}_{\nu} \sim Y_{\nu}$, and the contributions to $m_{L L}$ from the neutrinos $N_{1}^{c}, N_{2}^{c}$ are:

$$
\delta m_{L L}^{i j}=v_{2}^{2}\left(\frac{Y_{\nu}^{i 1} Y_{\nu}^{j 1}}{M_{R 1}}+\frac{Y_{\nu}^{i 2} Y_{\nu}^{j 2}}{M_{R 2}}\right)
$$

where

$$
M_{R 1} \sim \lambda^{\left|2 n_{1}+\sigma\right|}<\Sigma>, \quad M_{R 2} \sim \lambda^{\left|2 n_{2}+\sigma\right|}<\Sigma>
$$

and from Eq.36 $Y_{\nu}^{i p}=\lambda^{\left|l_{i}+n_{p}\right|}$. Similar to Eq.54 in this case we require

$$
\frac{\delta m_{L L}^{33}}{m_{L L}^{33}} \sim \frac{\lambda^{2\left|l_{3}+n_{1}\right|}}{\lambda^{2\left|l_{3}+n_{3}\right|}} \frac{M_{R 3}}{M_{R 1}}+\frac{\lambda^{2\left|l_{3}+n_{2}\right|}}{\lambda^{2\left|l_{3}+n_{3}\right|}} \frac{M_{R 3}}{M_{R 2}} \sim \lambda^{2}
$$

Thus the conditions for the "diagonal dominated case" are:

$$
\begin{aligned}
& 2\left|l_{3}+n_{1}\right|-2\left|l_{3}+n_{3}\right|+\left|2 n_{3}+\sigma\right|-\left|2 n_{1}+\sigma\right| \geq 2, \\
& 2\left|l_{3}+n_{2}\right|-2\left|l_{3}+n_{3}\right|+\left|2 n_{3}+\sigma\right|-\left|2 n_{2}+\sigma\right| \geq 2
\end{aligned}
$$

where at least one of the inequalities must be saturated. 
In the "off-diagonal dominated" case $M_{R R}$ can again be simply inverted leading to

$$
\delta m_{L L}^{i j}=v_{2}^{2}\left(\frac{Y_{\nu}^{i 1} Y_{\nu}^{j 2}}{M_{R 12}}+\frac{Y_{\nu}^{i 2} Y_{\nu}^{j 1}}{M_{R 12}}\right)
$$

where

$$
M_{R 12} \sim \lambda^{\left|n_{1}+n_{2}+\sigma\right|}<\Sigma>
$$

Again similar to Eq.54 we require

$$
\frac{\delta m_{L L}^{33}}{m_{L L}^{33}} \sim \frac{\lambda^{\left|l_{3}+n_{1}\right|+\left|l_{3}+n_{2}\right|}}{\lambda^{2\left|l_{3}+n_{3}\right|}} \frac{M_{R 3}}{M_{R 12}} \sim \lambda^{2}
$$

Thus the condition for the "off-diagonal dominated case" is:

$$
\left|l_{3}+n_{1}\right|+\left|l_{3}+n_{2}\right|-2\left|l_{3}+n_{3}\right|+\left|2 n_{3}+\sigma\right|-\left|n_{1}+n_{2}+\sigma\right|=2 .
$$

In the "democratic" case $M_{R R}$ can be readily inverted leading to a result of order

$$
\delta m_{L L}^{i j} \sim v_{2}^{2}\left(\frac{Y_{\nu}^{i 1} Y_{\nu}^{j 1}}{M}\right)
$$

where the right-handed neutrino masses in the upper block, $M$, are all equal by the democratic assumption and we have specialised to $n_{1}=n_{2}$ which implies from Eq.36 that $Y_{\nu}^{i 1} \sim Y_{\nu}^{i 2}$. Once again similar to Eq.54 we require

$$
\frac{\delta m_{L L}^{33}}{m_{L L}^{33}} \sim \frac{\lambda^{2\left|l_{3}+n_{1}\right|}}{\lambda^{2\left|l_{3}+n_{3}\right|}} \frac{M_{R 3}}{M} \sim \lambda^{2}
$$

Thus the condition for the "democratic case" is:

$$
2\left|l_{3}+n_{1}\right|-2\left|l_{3}+n_{3}\right|+\left|2 n_{3}+\sigma\right|-\left|2 n_{1}+\sigma\right|=2 \text {. }
$$

In practice examples of all three kinds can easily be constructed along the same lines as the explicit $Z=2$ case. The "democratic" case with $n_{1}=n_{2}$ is isomorphic to the $Z=2$ case. The $Z=2$ results trivially generalise in this case to $n_{p}=$ $\left(n_{2}, n_{2}, n_{3}\right)$ where some examples of charges were listed in Table 1. For example $l_{3}=-1 / 2, n_{p}=(0,0,1), \sigma=0$ satisfies all the "democratic" conditions with $a=2$ 
and $Y_{\nu}$ involving half-integer exponents. Clearly in the "democratic" case the $Z=2$ results can immediately be generalised to any number of right-handed neutrinos $Z$ with $n_{p}=\left(n_{2}, \ldots, n_{2}, n_{3}\right)$, where $\left(n_{2}, n_{3}\right)$ are the $Z=2$ charges.

The "diagonal dominated" case also follows a similar pattern to the $Z=2$ case with the lighter of $N_{1}^{c}, N_{2}^{c}$ playing the role of the subdominant right-handed neutrino in the $Z=2$ case. It is straightforward to scan over all the half-integer and integer charges which satisfy the "diagonal dominated" conditions and generate a list of charges for this case, analagous to Table 1 . A single example will suffice: $l_{3}=$ $-3 / 2, n_{p}=(0,1,2), \sigma=0$ satisfies all the "diagonal dominated" conditions and Eq.68 is saturated by $N_{2}^{c}$ which plays the role of the subdominant right-handed neutrino of the $Z=2$ case, with $N_{1}^{c}$ being both heavier and having more suppressed Dirac couplings. Again the "diagonal dominated" case can immediately be generalised to any number $Z$ of right-handed neutrinos $n_{p}=\left(n_{q}, n_{2}, n_{3}\right)$, where $\left(n_{2}, n_{3}\right)$ are the $Z=2$ charges with $N_{2}^{c}$ playing the role of the subdominant right-handed neutrino and $N_{q}^{c}$ giving subsubdominant contributions to the 23 block of $m_{L L}$.

Examples of the "off-diagonal dominated" kind have already been proposed in the literature, although they were not interpreted as being due to SRHND [15]. To show that the models in ref.[15] are examples of SRHND of the "off-diagonal dominated" kind it suffices to consider a specific example:

$$
l_{i}=(2,0,0), \quad n_{p}=(1,-1,0), \quad \sigma=0
$$

It is immediately clear that the charges in Eq.76 satisfy the conditions for SRHND in general Eq.43 and in particular the "off-diagonal dominated" conditions $\left|n_{1}+n_{2}+\sigma\right|<$ $\left|2 n_{1}+\sigma\right|,\left|2 n_{2}+\sigma\right|$ and Eq.72. This immediately substantiates our claim that these models correspond to SRHND of the "off-diagonal dominated" kind. Note that $Y^{\nu}$ involves integer exponents. In view of the interest in this example in the literature we develop it in a little more detail below. 
The charges in Eq.76 lead to the following neutrino Yukawa and heavy Majorana matrices

$$
Y^{\nu} \sim\left(\begin{array}{ccc}
\lambda^{3} & \lambda & \lambda^{2} \\
\lambda & \lambda & 1 \\
\lambda & \lambda & 1
\end{array}\right), \quad M_{R R} \sim\left(\begin{array}{ccc}
\lambda^{2} & 1 & \lambda \\
1 & \lambda^{2} & \lambda \\
\lambda & \lambda & 1
\end{array}\right)<\Sigma>
$$

Due to the assumed charges, the heavy Majorana matrix is dominated by three equal mass terms $\left\langle\Sigma>N_{1}^{c} N_{2}^{c},\left\langle\Sigma>N_{2}^{c} N_{1}^{c}\right.\right.$ and $\left\langle\Sigma>N_{3}^{c} N_{3}^{c}\right.$, leading to three roughly degenerate right-handed neutrinos. However of the three right-handed neutrinos it is $N_{3}^{c}$ which couples dominantly to the left-handed neutrinos of the second and third family, due to the assumed choice of $X$ charges, and hence dominates the 23 sector of $m_{L L}$. To see this we evaluate $m_{L L}$ in the basis in which

$$
M_{R R} \sim\left(\begin{array}{ccc}
0 & 1 & 0 \\
1 & 0 & 0 \\
0 & 0 & 1
\end{array}\right)<\Sigma>, \quad M_{R R}^{-1} \sim\left(\begin{array}{ccc}
0 & 1 & 0 \\
1 & 0 & 0 \\
0 & 0 & 1
\end{array}\right)<\Sigma>^{-1}
$$

In this basis we define $\tilde{Y}_{\nu} \equiv Y_{\nu} \Omega_{R R}^{\dagger}$ where

$$
\Omega_{R R} \sim\left(\begin{array}{lll}
1 & \lambda^{2} & \lambda \\
\lambda^{2} & 1 & \lambda \\
\lambda & \lambda & 1
\end{array}\right)
$$

Evaluating $m_{L L}$ in this basis we find from Eqs.9 and 78

$$
m_{L L}^{i j}=\frac{v_{2}^{2}}{<\Sigma>}\left(\tilde{Y}_{\nu}^{i 1} \tilde{Y}_{\nu}^{j 2}+\tilde{Y}_{\nu}^{i 2} \tilde{Y}_{\nu}^{j 1}+\tilde{Y}_{\nu}^{i 3} \tilde{Y}_{\nu}^{j 3}\right)
$$

corresponding to the contributions from the inverse mass terms $<\Sigma>N_{1}^{c} N_{2}^{c}$, $<$ $\Sigma>N_{2}^{c} N_{1}^{c}$ and $\left\langle\Sigma>N_{3}^{c} N_{3}^{c}\right.$, respectively. Since $\tilde{Y}_{\nu} \sim Y_{\nu}$ with the order one contributions to $\tilde{Y}_{\nu}$ coming exclusively from $N_{3}^{c}$, it is clear (by explicit evaluation of Eq.80) that $N_{3}^{c}$ dominates the contributions to the 23 block of $m_{L L}$, with corrections of order $\lambda^{2}$ coming from the other contributions. The remaining parts of $m_{L L}$ receive contributions at the same order as the $N_{3}^{c}$ contributions coming from $N_{1}^{c}, N_{2}^{c}$. Thus the resulting light effective neutrino matrix is as in Eq.20, with SRHND in the 23 sector due to $N_{3}^{c}$ dominance with $O\left(\lambda^{2}\right)$ corrections from other right-handed neutrinos.

Finally we note that for $Z>3$ the above three categories "diagonal dominated", "off-diagonal dominated" and "democratic" may be combined in all possible ways. 


\section{Conclusion}

We have suggested a natural explanation of both neutrino mass hierarchies and large neutrino mixing angles, as required by the atmospheric neutrino data, in terms of a single right-handed neutrino giving the dominant contribution to the 23 block of the light effective neutrino matrix. We illustrated this mechanism in the framework of models with a single pseudo-anomalous $U(1)_{X}$ family symmetry, expanding all masses and mixing angles in terms of the Wolfenstein parameter $\lambda$. Sub-dominant contributions to the 23 sector from other right-handed neutrinos, suppressed by a factor of $\lambda^{2}$, are required to give small mass splittings appropriate to the small angle MSW solution to the solar neutrino problem. We gave general conditions for achieving this in the framework of $U(1)_{X}$ family symmetry models containing arbitrary numbers of right-handed neutrinos $Z$. We classified the $Z=3$ cases into three categories: "diagonal dominated", "off-diagonal dominated" and "democratic", and discussed

examples of each kind. Although the approach in [15] is based on the formal condition that the subdeterminant vanishes to order $\lambda^{2}$, we have shown that explicit examples of this kind of model may be classified within our framework as SRHND of the "offdiagonal dominated" kind. Although we discussed a particular family symmetry it is clear that the idea of SRHND is more general and has recently been used in a model with $S U(2)$ family symmetry [16].

\section{References}

[1] Y. Fukuda et al., Super-Kamiokande collaboration, Phys. Lett. B433 (1998) 9; Phys. Lett. B436 (1998) 33; Phys. Rev. Lett. 81 (1998) 1562.

[2] S. Hatakeyama et al., Kamiokande collaboration, Phys. Rev. Lett.81 (1998) 2016; M. Ambrosio et al., MACRO collaboration, Phys. Lett. B434 (1998) 451. 
[3] K.S. Hirata et al., Kamiokande collaboration, Phys. Lett. B205 (1988) 416, Phys. Lett. B280 (1992) 146; E.W. Beier et al., Phys. Lett. B283 (1992) 446; Y. Fukuda et al., Kamiokande collaboration, Phys. Lett. B335 (1994) 237; K. Munakata et al., Kamiokande collaboration, Phys. Rev. D56 (1997) 23; Y. Oyama et al., Kamiokande collaboration, hep-ex/9706008; D. Casper et al., IMB collaboration, Phys. Rev. Lett. 66 (1991) 2561; R. Becker-Szendy et al., IMB collaboration, Phys. Rev. D46 (1992) 3720; W.W.M. Allison et al., Soudan-2 collaboration, Phys. Lett. B391 (1997) 491.

[4] Y. Fukuda et al, Super-Kamiokande Collaboration, hep-ex/9805021. B.T. Cleveland Nucl. Phys. B38 (1995) 47; Gallex Collaboration, Phys. Lett. B388 (1996) 384; Gallex Collaboration, TAUP97, Laboratori Nazionali del Gran Sasso September 1997, to appear on Nucl. Phys. B (Proc. Suppl.); V. Gavrin et al (SAGE Collaboration) Phys. Lett. B328 (1994) 234; SAGE Collaboration, Neutrino 96, Helsinki June 1996 to appear on Nucl. Phys. B (Proc. Suppl.).

[5] L. Wolfenstein, Phys. Rev. D17 (1978) 2369;

S. Mikheyev and A. Yu. Smirnov, Sov. J. Nucl. Phys. 42 (1985) 913.

[6] V.N. Gribov, B.M. Pontecorvo, Phys. Lett. B 28 (1969) 493.

[7] more recently, see, e.g. V. Barger, K. Whisnant, R.J.N. Phillips, Phys. Rev. D24 (1981) 538; S. L. Glashow, L. M. Krauss, Phys. Lett. 190B (1987) 199; V. Barger, R.J.N. Phillips, K. Whisnant, Phys. Rev. Lett. 65 (1990) 3084, ibid 69 (1992) 3135; N.Hata, P.G. Langacker, Phys. Rev. D 56 (1997) 6107; P.I. Krastev, S.T. Petcov, Nucl. Phys. B 449 (1995) 605. S. L. Glashow, P. J. Kernan, L. M. Krauss, hep-ph/9808470.

[8] J.N. Bahcall, P.I. Krastev and A.Y. Smirnov, Phys. Rev. D58 (1998) 096016; and references therein. 
[9] M. Gell-Mann, P. Ramond and R. Slansky in Sanibel Talk, CALT-68-709, Feb 1979, and in Supergravity (North Holland, Amsterdam 1979);

T. Yanagida in Proc. of the Workshop on Unified Theory and Baryon Number of the Universe, KEK, Japan, 1979.

[10] I. Sogami et al, hep-ph/9807449; K. Akama and K. Katsuura, hep-ph/9807534; M. Drees et al, Phys. Rev. D57 (1998) 5335; A. Joshipura and A. Smirnov, Phys. Lett. B439 (1998) 103; V. Barger, S.Pakvasa, T.J. Weiler and K. Whisnant, Phys. Lett. B437 (1998) 107; R. Barbieri, L.J. Hall, D. Smith, A. Strumia and N. Weiner, hep-ph/9807235; G. Lazarides and N. Vlachos, Phys. Lett. B441 (1998) 46; M. Tanimoto, Phys. Rev. D59 (1999) 017304; M. Gonzalez-Garcia, H. Nunokawa, O.L.G. Perez and J.W.F. Valle, hep-ph/9807305; M. Jezabek and Y. Sumino, Phys. Lett. B440 (1998) 327; J. Pati, hep-ph/9807315; V. Barger, T. Weiler and K. Whisnant, Phys. Lett. B440 (1998) 1; E. Ma, Phys. Lett. B442 (1998) 238; S. M. Bilenky, C. Giunti and W. Grimus, hep-ph/9812360; G.L. Fogli, E. Lisi, A. Marrone and G. Scioscia, Phys. Rev. D59 (1999) 033001; R. Barbieri, L.J. Hall and A. Strumia, hep-ph/9808333; H. Fritzsch, Z. Xing, hep-ph/9807234; S.M. Bilenky, C. Giunti, hep-ph/9802201; M. Tanimoto, hep-ph/9807517; R. Mohapatra and S. Nussinov, hep-ph/9808301. M. Fukugita, M. Taminoto and T. Yanagida, hep-ph/9809554; W. Buchmuller and T. Yanagida, hep-ph/9810308; Y. Grossman, Y. Nir, Y. Shadmi, JHEP 9810 (1998) 007; C.D. Froggatt, M. Gibson and H.B. Nielsen, hep-ph/9811265; Z. Berezhiani and A. Rossi, hepph/9811447; C. Wetterich, hep-ph/9812426. S. Lola and J. Vergados, Progress in Particle and Nuclear Physics 40 (1998) 71; V. Barger, S. Pakvasa, T. Weiler and K. Whisnant, hep-ph/9806387; A. Baltz, A.S. Goldhaber, M. Goldhaber, hep-ph/9806540; M. Jezabek and Y. Sumino, hep-ph/9807310; Y. Nomura and T. Yanagida, hep-ph/9807325; H. Georgi and S. Glashow, hep-ph/9808293; K.S. Babu, J.C. Pati and F. Wilczek, hep-ph/9812538; F. Wilczek, hep-ph/9812538; 
R. Barbieri, L.J. Hall, G.L. Kane and G.G. Ross, hep-ph/9901228; B.C. Allanach, hep-ph/9806294; Q. Shafi and Z. Tavartkiladze, hep-ph/9811282; V. Barger et al., Phys. Rev. D58 (1998) 093016; M. Bando and K. Yoshioka, hep-ph/9806400; C.H. Albright and S.M. Barr, Phys. Rev. D58 (1998) 013002; hep-ph/9901318; C.H. Albright, K.S. Babu and S.M. Barr, Phys. Rev. Lett. 81 (1998) 1167; J. Ellis, G. Leontaris, S. Lola and D.V. Nanopoulos, hep-ph/9808251.

[11] S. F. King, Phys. Lett. B439 (1998) 350, hep-ph/9806440.

[12] S. Davidson and S. F. King, Phys. Lett. B445 (1998) 191, hep-ph/9808296.

[13] S. Lola and G.Ross, hep-ph/9902283; G. Leontaris, S. Lola and G . Ross, Nucl. Phys. B454 (1995) 25; H. Dreiner, G. Leontaris, S. Lola G. Ross and C. Scheich, Nucl. Phys. B436 (1995) 461.

[14] J. Elwood, N. Irges and P. Ramond, Phys. Rev. Lett. 81 (1998) 5064, hepph/9807228; N. Irges, S. Lavignac and P. Ramond, Phys. Rev. D58 (1998) 035003, hep-ph/9802334.

[15] G. Altarelli and F. Feruglio, hep-ph/9807353, G. Altarelli and F. Feruglio, hepph/9809596, G. Altarelli and F. Feruglio, hep-ph/9812475.

[16] R. Barbieri, P. Creminelli and A. Romanino, hep-ph/9903460.

[17] Z. Maki, M. Nakagawa and S. Sakata, Prog. Theo. Phys. 28 (1962) 247.

[18] L. Wolfenstein, Phys. Rev. Lett 51 (1983) 1945.

[19] M. Apollonio et al hep-ex/9711002.

[20] C. D. Froggatt and H. B. Nilsen, Nucl. Phys. B147 (1979) 277.

[21] H. Fritzsch, Phys. Lett. 70B (1977) 436; B73 (1978) 317; Nucl. Phys. B155 (1979) 189; J. Harvey, P. Ramond and D. Reiss, Phys. Lett. B92 (1980) 309; 
S. Dimopoulos, L. J. Hall and S. Raby, Phys. Rev. Lett. 68 (1992) 1984; C. Wetterich, Nucl. Phys. B261 (1985) 461; P. Kaus and S. Meshkov, Mod. Phys. Lett. A3 (1988) 1251.

[22] L. Ibanez and G.G. Ross, Phys. Lett. B332 (1994) 100.

[23] V. Jain and R. Shrock, Phys. Lett. B352 (1995) 83, hep-ph/9412367.

[24] P. Binetruy and P. Ramond, Phys. Lett. B350 (1995) 49, hep-ph/9412385.

[25] M. Green and J. Schwartz, Phys. Lett. B149 (1984) 117.

[26] A. Rasin and J. P. Silva, Phys. Rev. D49 (1994) 20, hep-ph/9309240; P. Ramond, hep-ph/9809401. 